\title{
Random Integral Equation of the Volterra Type with Applications
}

\author{
Hamdin Ahmed Alafif \\ Khartoum University, Faculty of Education, Omdurman, Sudan \\ Email: hamdin@163.com
}

Received December 2013

\section{Abstract}

In this paper we shall present some of the most general results which have been obtained to date concerning random integral equations of the Volterra type. Some results of Tsokos [4] are given for the random integral equation

$$
x(t ; w)=h(t ; w)+\int_{0}^{t} k(t, \tau ; w) f(\tau, x(\tau ; w)) d \tau
$$

where $t \geq 0$ and 1$) w$ is appoint of $\Omega ; 2) h(t ; w)$ is the stochastic free term or free random variable defined for $0 \leq t$ and $w \in \Omega ; 3) \quad x(t ; w)$ is the unknown random variable for each $t \geq 0$ 4) the stochastic kernel $k(t, \tau ; w)$ is defined for $0 \leq \tau \leq t<\infty$ and $w \in \Omega$.

\section{Keywords}

Volterra Integral, Banach Space, Asymptotic Stability, Poincare-Lyapunov, Telephone Traffic Theory

\section{Introduction}

In Section 2, we present some results of [4] for the random integral equation in Section 2, investigating the existence and uniqueness of random solution of Equation (1.1). The asymptotic behavior of the random solution and its stability properties are also considered. In Section 3 some applications of Equation (1.1) will be presented in the areas of telephone traffic theory and a generalization of the classical poincare'-Lyapunov theorem [3]

Remark Below are some spaces which we use in our study

a) $C=C\left(\mathbb{R}_{+}, L_{2}(\Omega, \mathcal{F}, P)\right)$ denote the space of all continuous and bounded functions on $\mathbb{R}_{+}$with values in $L_{2}(\Omega, \mathcal{F}, P)$.

b) $C_{g}=C_{g}\left(\mathbb{R}_{+}, L_{2}(\Omega, \mathcal{F}, P)\right)$ the space of all continuous functions from $\mathbb{R}_{+}$into $L_{2}(\Omega, \mathcal{F}, P)$ such that

$$
\left\{\int_{\Omega}|x(t ; w)|^{2} d P(w)\right\}^{\frac{1}{2}} \leq z g(t) \quad t \in \mathbb{R}_{+}
$$

where $Z$ is a positive number and $g(t)$ is a positive continuous function defined on $\mathbb{R}_{+}$

(c) $C_{C}=C_{C}\left(\mathbb{R}_{+}, L_{2}(\Omega, \mathcal{F}, P)\right)$ is the space of all continuous functions from $\mathbb{R}_{+}$into $L_{2}(\Omega, \mathcal{F}, P)$ with 
the topology of uniform convergence on the intervals $[0, T]$ for $T>0$.

Where $(\Omega, \mathcal{F}, P)$ a probability measure space.

\section{The Random Integral Equation}

$$
x(t ; w)=h(t ; w)+\int_{0}^{t} k(t, \tau ; w) f(\tau, x(\tau, w)) d \tau
$$

This equation seems to be more general than any random Volterra integral equation which has been studied to date. The generality consists primarily in the choice of the stochastic kernel. In this Section we shall investigate the existence of a random solution and its uniqueness and asymptotic behavior and shall consider a number of special cases as corollaries of the main theorems. Finally, the stability properties of the random solution will be investigated.

To accomplish our objectives here we employ certain aspects of the methods of "admissibility theory" which has been utilized quite recently in the theory of deterministic integral equations by Corduneanu [4].

\section{Existence and Uniqueness of a Random Solution}

Let $B$ and $D$ be a pair of Banach spaces and $T$ a linear operator. With respect to the study of this Section, we state the following Lemma which will be used in the main theorems.

Lemma 3.1 Let $T$ he a continuous operator from $C_{C}\left(\mathbb{R}_{+}, L_{2}(\Omega, \mathcal{F}, P)\right)$ into itself. If $B$ and $D$ are Banach spaces stronger than $C_{C}$ and the pair $(B, D)$ is admissible with respect to $T$, then $T$ is a continuous operator from $B$ to $D$.

\section{Proof}

First we will prove that the operator $T$ is closed form $B$ to $D$. Let us consider the sequence $x_{n}(t ; w) \in B$ such that $x_{n}(t ; w) \stackrel{B}{\longrightarrow} x(t, w)$ as $n \rightarrow \infty$. Let us assume $\left(T x_{n}\right)(t ; w) \stackrel{D}{\longrightarrow} y(t ; w)$ as $n \rightarrow \infty$. Now we must show that $(T x)(t ; w)=y(t ; w)$. Since $x_{n}(t, w) \rightarrow x(t, w)$ in $\mathrm{B}, x_{n}(t, w) \rightarrow x(t, w)$ in $C_{C}$. But since $T$ : $C_{c} \rightarrow C_{c}$ is continuous we have $\left(T x_{n}\right)(t ; w) \rightarrow(T x)(t ; w)$ in $C_{C}$. On the other hand $\left(T x_{n}\right)(t ; w) \rightarrow y(t ; w)$ in $D$, which implies that $\left(T x_{n}\right)(t ; w) \rightarrow y(t ; w)$ in $C_{C}$. Hence $(T x)(t ; w) \rightarrow y(t ; w)$ because the limit is unique in $C_{C}$. Therefore the operator $T$ is closed. Then by the closed-graph theorem it follows that $T$ is continuous operator from $B$ to $D$.

Remark since $T$ is closed and continuous linear operator, it is also bounded (Yosida [1], p. 10). Then it follows that we can find a constant $k>0$ such that

$$
\|(T x)(t ; w)\|_{D} \leq k\|x(t ; w)\|_{B}
$$

With respect to our aim here, we state and prove the following theorems.

Theorem 3.2 Let us consider (1.1) under the following conditions:

1) $B$ and $D$ are Banach spaces stronger than $C_{C}\left(\mathbb{R}_{+}, L_{2}(\Omega, \mathcal{F}, P)\right)$ such that $(B, D)$ is admissible with respect to the operator

$$
(T x)(t ; w)=\int_{0}^{t} k(t, \tau ; w) x(\tau ; w) d \tau
$$

2) $x(t ; w) \rightarrow f(t, x(t ; w))$ is a continuous operator on

$$
S=\left\{x(t ; w): x(t, w) \in D,\|x(t ; w)\|_{D} \leq \rho\right\}
$$

with values in $B$, also satisfying

$$
\|f(t, x(t ; w))-f(t, y(t ; w))\|_{B} \leq \lambda\|x(t ; w)-y(t ; w)\|_{D}
$$

with $x(t ; w), y(t ; w) \in S$ and $\lambda$ a constant.

3) $\|h(t ; w)\| \in D$

Then there exists a unique random solution of the random integral Equation (1.1), provided that

$$
\lambda<k^{-1},\|h(t ; w)\|_{D}+k\|f(t, 0)\|_{B} \leq \rho(1-\lambda k),
$$

where $k$ is the norm of the operator $T$. 


\section{Proof}

Let us define an operator $U$ on $S$ into $D$ as follows

$$
(U x)(t ; w)=h(t ; w)+\int_{0}^{t} k(t, \tau, w) f(\tau, x(\tau ; w)) d \tau
$$

Now we must show that $U$ is a contracting operator and $U(S) \subset S$. Consider a function $y(t ; w)$ in $S$. We can write

$$
(U y)(t ; w)=h(t ; w)+\int_{0}^{t} k(t, \tau ; w) f(\tau, y(\tau ; w)) d \tau
$$

Subtracting Equation (3.2) from Equation (3.1) we have

$$
(U x)(t ; w)-(U y)(t, w)=\int_{0}^{t} k(t, \tau ; w)[f(\tau, x(\tau ; w))-f(\tau, y(\tau ; w))] d \tau
$$

Since $U(S) \subset D$ and $D$ is a Banach space then

$$
(U x)(t, w)-(U y)(t, w) \in D
$$

By assumptions 1) and 2), $[f(\tau, x(\tau ; w))-f(\tau, y(\tau ; w))] \in B$. From Lemma 3.1 we have seen that $T$ is continuous operator from the Banach space $B$ into $D$ which implies that we can find a constert $k>0$ such that

$$
\|(T x)(t ; w)\|_{D} \leq k\|x(t ; w)\|_{B}
$$

That is $\|(U x)(t ; w)-(U y)(t ; w)\|_{D} \leq k \| f\left(t, x(t ; w)-f\left(t, y(t ; w) \|_{B}\right.\right.$

Now, applying Lipschitz's condition given in (ii) we have

$$
\|(U x)(t ; w)-(U y)(t ; w)\|_{D} \leq \lambda k\|x(t ; w)-y(t ; w)\|_{D}
$$

Using the condition that $\lambda k<1$, the operator $U$ is a contracting operator.

It now remains to be show that $U(S) \subset S$. For every function $x(t ; w) \in S$ we have

$$
(U x)(t ; w)=h(t ; w)+\int_{0}^{t} k(t, \tau ; w) f(\tau, x(\tau ; w))
$$

Applying condition 3) and Lemma(3.1) we can write expression(3.3) as follows

$$
\|(U x)(t ; w)\|_{D} \leq\|h(t ; w)\|_{D}+k\|f(t, x(t ; w))\|_{B} d \tau
$$

In (3.4), $\|f(t, x(t ; w))\|_{B}$ can be written as

$$
\|f(t, x(t ; w))\|_{B}=\|f(t, x(t ; w))-f(t, 0)+f(t, 0)\|_{B} \leq\|f(t, x(t ; w))-f(t, 0)\|_{B}+\|f(t, 0)\|_{B}
$$

Using Lipschitz's condition we have

$$
\|f(t, x(t ; w))\|_{B} \leq \lambda\|x(t, w)-0\|_{D}+\|f(t, 0)\|_{B}
$$

We can now write expression (3.4) as follows

$$
\|(U x)(t ; w)\|_{D} \leq\|h(t ; w)\|_{D}+k \lambda\|x(t ; w)\|_{D}+k\|f(t, 0)\|_{B}
$$

Since $x(t ; w) \in S$ and $\|x(t ; w)\|_{D} \leq \rho$, (3.5) can be written as

$$
\|(U x)(t ; w)\|_{D} \leq\|h(t ; w)\|_{D}+k \lambda \rho+k\|f(t, 0)\|_{B}
$$

Applying the condition of the theorem that

$$
\|h(t ; w)\|_{D}+k\|f(t, 0)\|_{B} \leq \rho(1-\lambda k)
$$

(3.6) becomes

$$
\|(U x)(t ; w)\|_{D} \leq \rho(1-\lambda k)+k \lambda \rho \text { or }\|(U x)(t ; w)\|_{D} \leq \rho
$$

Which implies that $(U x)(t ; w) \in S$ for all random variables $x(t ; w) \in S$ or $U(S) \subset S$. Therefore, since $U$ is a contracting operator and $U(S) \subset S$ applying Banach's fixed-point theorem.

There exists a unique random solution $x(t ; w) \in S$ such that 


$$
(U x)(t ; w)=h(t ; w)+\int_{0}^{t} k(t, \tau ; w) f(\tau, x(\tau ; w)) d \tau=x(t ; w)
$$

\section{Some Special Cases}

Now we shall derive some particular cases of theorem 3.2 choosing in a convenient manner the spaces $B$ and $D$. Consider that a $C_{g}$ space is a space of all continuous functions from $\mathbb{R}_{+} \rightarrow L_{2}(\Omega, \mathcal{F}, P)$ such that

$$
\|x(t ; w)\|=\left\{\int_{\Omega}|x(t ; w)|^{2} d P(w)\right\}^{\frac{1}{2}} \leq z g(t)
$$

where $t \in \mathbb{R}_{+}, \mathrm{Z}$ is a number grater than zero, and $g(t)$ is a continuous function greater than zero. Also $C_{C}\left(\mathbb{R}_{+}, L_{2}(\Omega, \mathcal{F}, P)\right)$ is the space of all continuous function from $\mathbb{R}_{+}$into $L_{2}(\Omega, \mathcal{F}, P)$ with the topology of uniform convergence on the interval $[0, T]$ for any $T>0$ and the norm of the stochastic kernel of the integral equation can be defined as follows:

$$
k(t, \tau)=\|k(t, \tau ; w)\|=P-e s s \sup |k(t, \tau ; w)|
$$

With respect to $w \in \Omega$. That is

$$
\|k(t, \tau ; w)\|=\inf _{\Omega_{0}}\left\{\sup _{\Omega-\Omega_{0}}|k(t, \tau ; w)|\right\}
$$

With $P\left(\Omega_{0}\right)=0$.

Theorem 4.1 Let us consider the random integral Equation (1.1) under the following conditions

1) there exist a number $A>0$ and a continuous function $g(t)>0$ such that

$$
\int_{0}^{t}\|k(t, \tau ; w)\| g(\tau) \leq A, \quad t \in \mathbb{R}_{+}
$$

2) $f(t, x)$ is a continuous vector-valued function for $t \in \mathbb{R}_{+},\|x(t ; w)\| \leq \rho$ such that

$$
f(t, 0) \in C_{g},\|f(t, x(t ; w))-f(t, y(t ; w))\| \leq \lambda g(t)\|x(t ; w)-y(t ; w)\|
$$

3) $h(t, w)$ is a continuous bounded function on $\mathbb{R}_{+}$whose values are in $L_{2}(\Omega, \mathcal{F}, P)$. Then there exists a unique random solution $x(t ; w) \in C$ of the random integral Equation (1.1) such that

$$
\|x(t ; w)\|_{C}=\sup _{0 \leq t}\left\{\int_{\Omega}|x(t ; w)|^{2} d P(w)\right\}^{\frac{1}{2}} \leq \rho
$$

for $t \in \mathbb{R}_{+}$as long as $\|h(t ; w)\|, \lambda$ and $\|f(t, 0)\|_{C}$ are small enough.

Proof We must show that under condition (i) of the theorem the pair of Banach spaces $\left(C_{g}, C\right)$ is admissible. That is $\left(C_{g}, C\right)$ is admissible with respect to the integral operator

$$
(T x)(t ; w)=\int_{0}^{t} k(t, \tau ; w) x(\tau, w) d \tau
$$

for $x(t ; w) \in C_{g}$ we have

$$
(T x)(t ; w)=\int_{0}^{t} k(t, \tau ; w) x(\tau ; w) d \tau
$$

or

$$
\|(T x)(t ; w)\| \leq \int_{0}^{t}\|k(t, \tau ; w) x(\tau ; w)\| d \tau \leq \int_{0}^{t}\|k(t, \tau ; w)\|[\|x(\tau ; w)\| / g(\tau)] g(\tau) d \tau
$$

where $\|k(t, \tau ; w)\|=P-e s s \sup _{w}|k(t, \tau ; w)|$ is a function only of $(t, \tau)$. Using the definition of the norm in $C_{g}$, that is

$$
\sup _{0 \leq t}\left\{\left\{\int_{\Omega}|x(t ; w)|^{2} d P(w)\right\}^{\frac{1}{2}}[1 / g(t)]\right\}=\|x(t ; w)\|_{C_{g}}
$$

For $g(t)>0$ we can write (4.1) as follows 


$$
\|(T x)(t ; w)\| \leq \int_{0}^{t}\|k(t, \tau ; w)\|\|x(\tau ; w)\|_{C_{g}} g(\tau) d \tau \leq\|x(t ; w)\|_{C_{g}} \int_{0}^{t}\|k(t, \tau ; w)\| g(\tau) d \tau \leq A\|x(\tau ; w)\|_{C_{g}}
$$

Therefore $\|(T x)(t ; w)\|$ is bounded and hence $(T x)(t ; w) \in C$ for all $(T x)(t ; w)$, hence $T C_{g} \subset C$, which implies that the pair of Banach spaces $\left(C_{g}, C\right)$ is admissible with respect to the integral operator as defined here.

The remainder of the proof is analogous to that of theorem 3.2 and is omitted.

For the special case where $g(t)=1$ we state and prove the following corollary 4.2 let us assume that the random integral Equation (1.1) satisfies the following conditions:

1) $\int_{0}^{t}\|k(t, \tau ; w)\| d \tau \leq A, \quad t \in \mathbb{R}_{+}$, where $A$ is a constant greater than zero.

2) $f(t, x)$ is a continuous function from $\mathbb{R}_{+}$into $\mathbb{R}$ uniformly in $x$ such that

$$
|f(t, x)-f(t, y)| \leq \lambda|x-y|
$$

3) $h(t ; w)$ is a continuous bounded function from $\mathbb{R}_{+}$into $L_{2}(\Omega, \mathcal{F}, P)$. Then there exists a unique bounded random solution on $\mathbb{R}_{+}$of the random integral Equation (1.1) if $\lambda$ is small enough.

Proof We must show that under condition (i) of the corollary the pair of Banach spaces $(C, C)$ is admissible. For a function $x(t ; w) \in C$ we have

$$
(T x)(t ; w)=\int_{0}^{t} k(t, \tau ; w) x(\tau ; w) d \tau
$$

or

$$
\|(T x)(t ; w)\| \leq \int_{0}^{t}\|k(t, \tau ; w)\|\|x(\tau ; w)\| d \tau
$$

Applying the definition of the norm as used in theorem 4.1, in equality (4.2) can be written as follows

$$
\|(T x)(t ; w)\| \leq\|x(t ; w)\| \int_{0}^{t}\|k(t, \tau ; w)\| d \tau \leq A\|x(t ; w)\|, t \in \mathbb{R}_{+}
$$

Therefore $(T x)(t ; w) \in C$ for every random variable $x(t ; w)$ or $T C \subset C$, which implies that $(C, C)$ is admissible. The remainder of the proof follows from theorem 3.2

The following two corollaries are particular cases of theorem 4.1.

Corollary 4.3 Assume that the random integral equation (1.1) satisfies the following conditions:

1) $\|k(t, \tau ; w)\| \leq \Lambda_{1}$, for $0 \leq \tau \leq t<\infty$ and $\int_{0}^{\infty} g(t) d t<\infty$

2) Same condition as in theorem 4.1, condition 2).

3) Same condition as in theorem 4.1, condition 3).

Then there exists a uniqe random solution of Equation (1.1) bounded on $\mathbb{R}_{+}$if $\|h(t ; w)\|, \lambda$ and $\|f(t, 0)\|$ are sufficiently small.

Proof It is only necessary to show that the pair of Banach spaces $\left(C_{g}, C\right)$ is admissible with respect to the integral operator

$$
(T x)(t ; w)=\int_{0}^{t} k(t, \tau ; w) x(\tau ; w) d \tau
$$

Along with condition (1) of the corollary. For a function $x(t ; w) \in C_{g}$ expression (4.3) implies that

$$
\|(T x)(t ; w)\| \leq \int_{0}^{t}\|k(t, \tau ; w)\|\|x(\tau ; w)\| d \tau
$$

Applying hypothesis (i) of the corollary, we have

$$
\|(T x)(t ; w)\| \leq \Lambda_{1} \int_{0}^{t}\|x(\tau ; w)\| d \tau \leq \Lambda_{1} \int_{0}^{t}[\|x(t ; w)\| / g(\tau)] g(\tau) d \tau
$$

Utilizing the definition of norm as applied in theorem 4.5 to in equality (4.5) we have

$$
\|(T x)(t ; w)\| \leq \Lambda_{1}\|x(t ; w)\|_{C_{g}} \int_{0}^{t} g(\tau) d \tau
$$


but applying condition (1) of the corollary (4.6) is written as

$$
\|(T x)(t ; w)\| \leq M \text { for all } t \geq 0
$$

Therefore the function $x(t ; w) \in C_{g}$ implies that $(T x)(t ; w) \in C$ or $T C_{g} \subset C$. Hence the pair $\left(C_{g}, C\right)$ is admissible, and, since condition (2) and (3) are the same as in theorem 4.1 the proof is complete.

Corollary 4.4 Let us consider the random integral Equation (1.1) under the following conditions

1) $\|k(t, \tau ; w)\| \leq \Lambda_{2} e^{-\alpha(t-\tau)}$, for $0 \leq \tau \leq t<+\infty$, and

$$
\sup _{t \in \mathbb{R}_{+}}\left\{\int_{t}^{t+1} g(\tau) d \tau\right\}<\infty
$$

where $\Lambda_{2}$ and $\alpha$ are positive numbers.

2) same as condition (2) of theorem 4.1.

3) same as condition (3) of theorem 4.1.

Then there exists a unique random solution of the random integral Equation (1.1) bounded on $\mathbb{R}_{+}$if $\|h(t ; w)\|, \lambda$ and $\|f(t, 0)\|$ are small enough.

\section{Asymptotic Stability of the Random Solution}

With respect to the asymptotic behavior of the random solution of the stochastic integral Equation (1.1), we state and prove the following theorem, the objective of which is to investigate the possibility of the random solution being asymptotically exponentially stable.

Theorem 5.1 Let us consider the stochastic integral Equation (1.1) under the following conditions

1) $\|k(t, \tau ; w)\| \leq \Lambda_{2} e^{-\alpha(t-\tau)}$, for $0 \leq \tau \leq t<+\infty, \Lambda_{2}>0$, and $\alpha>0$

2) $f(t, x)$ is a continuous function from $\mathbb{R}_{+} \times \mathbb{R}$ into $\mathbb{R}$ such that

$$
f(t, 0)=0 \text { and }|f(t, x)-f(t, y)| \leq \lambda|x-y|
$$

3) $\|h(t ; w)\| \leq \rho e^{-\beta t}$ where $\rho$ and $\beta$ are positive numbers such that $0<\beta<\alpha$.

Then there exists a unique random solution of Equation (2.1.1) such that

$$
\left\{\int_{\Omega}|x(t ; w)|^{2} d P(w)\right\}^{\frac{1}{2}} \leq \rho e^{-\beta t} \quad t \in \mathbb{R}_{+}
$$

as long as $\lambda$ is small enough.

proof We must show that the pair of Banach spaces $\left(C_{g}, C_{g}\right)$ with $g(t)=e^{-\beta t}$, is admissible under conditions (1) and (2) of the theorem.

That is $\left(C_{g}, C_{g}\right)$ is admissible with respect to the operator defined by

$$
(T x)(t ; w)=\int_{0}^{t} k(t, \tau ; w) x(\tau ; w) d \tau
$$

The norm of the expression (5.1) can be written as

$$
\|(T x)(t ; w)\| \leq \int_{0}^{t}\|k(t, \tau ; w)\|\|x(\tau ; w)\| d \tau
$$

Applying condition (1) of the theorem, we have

$$
\|(T x)(t ; w)\| \leq \Lambda_{2} \int_{0}^{t} e^{-\alpha(t-\tau)}\|x(t ; w)\| d \tau
$$

Hence

$$
\|(T x)(t ; w)\| \leq \Lambda_{2} \int_{0}^{t} e^{-\alpha(t-\tau)}[\|x(\tau ; w)\| / g(\tau)] g(\tau) d \tau
$$

Using the definition of the norm on the $C_{g}$ space, inequality (5.4) can be written as

$$
\begin{aligned}
& \|(T x)(t ; w)\| \leq M \int_{0}^{t} e^{-\alpha(t-\tau)} g(\tau) d \tau=M \int_{0}^{t} e^{-\alpha(t-\tau)} e^{-\beta \tau} d \tau \\
& =M e^{-\alpha t}[1 /(\alpha-\beta)]\left(e^{(\alpha-\beta) t}-1\right)=M(\alpha-\beta)^{-1}\left(e^{-\beta t}-e^{-\alpha t}\right)
\end{aligned}
$$


Since $0<\beta<\alpha$, we can majorize inequality (5.5) as follows

$$
\|(T x)(t ; w)\| \leq M(\alpha-\beta)^{-1}\left(e^{-\beta t}-e^{-\alpha t}\right)<M(\alpha-\beta)^{-1} e^{-\beta t}, \quad t \in \mathbb{R}_{+}
$$

Which implies that $(T x)(t ; w) \in C_{g}$ for a function $x(t ; w) \in C_{g}$. Therefore the pair of Banach spaces $\left(C_{g}, C_{g}\right)$ is admissible with respect to the operator $T$ where $g(t) \stackrel{g}{=} e^{-\beta t}$. Condition (3) of the theorem means that $h(t ; w) \in C_{g}$.

Applying condition (2), we have

$$
\|f(t, x(t ; w))-f(t, y(t ; w))\|_{C_{g}} \leq \lambda\|x(t ; w)-y(t ; w)\|_{C_{g}}
$$

Hence, all the conditions of theorem 4.1 have been satisfied, which implies that there exists a unique random solution of the integral Equation (1.1) such that

$$
\left\{\int_{\Omega}|x(t ; w)|^{2} d P(w)\right\}^{\frac{1}{2}} \leq \rho e^{-\beta t}
$$

Remark It is now clear that under these conditions there exists a random solution of the random integral Equation (1.1) which is exponentially asymptotically stable that is

$$
\lim _{t \rightarrow \infty}\left\{\int_{\Omega}|x(t ; w)|^{2} d P(w)\right\}^{\frac{1}{2}}=0
$$

\section{Some Applications of the Equation}

$$
x(t ; w)=h(t ; w)+\int_{0}^{t} k(t, \tau ; w) f(\tau, x(\tau ; w)) d \tau
$$

In this Section we shall present some applications of the results of the previous Section. We shall first consider a generalization of the classical stability theorem of Poincaré and Lyapunov. We shall then study a stochastic integral equation arising in the theory of telephone traffic, arelated study of which was done by Fortet [1].

\subsection{Generalization of Poincaré-Lyapunov Stability Theorem}

As an example to illustrate our results, we shall generalized the classical stability theorem of Poincaré and Lyapunov(Tsokos [3]). That is consider the following random differential system

$$
\dot{x}(t ; w)=A(w) x(t ; w)+f(t, x(t ; w)) \quad t \geq 0
$$

where 1) $x(t ; w)$ is the unknown $n \times 1$ random vector; 2) $A(w)$ is an $n \times n$ matrix whose elements are measurable functions; and 3) $f(t, x)$ is, for $t \in \mathbb{R}_{+}$and $x \in \mathbb{R}$, an $n \times 1$ vector-valued function.

Now we shall reduce the random differential system (6.1) to astochastic integral equation which will be a special case of the stochastic integral Equation (1.1). Myltiplying the random system (6.1) by $e^{-A(w) t}$, we have

$$
e^{-A(w) t} \dot{x}(t ; w)-A(w) e^{-A(w) t} x(t ; w)=e^{-A(w) t} f((t, x(t ; w))
$$

But $(d / d t)\left\{e^{-A(w) t} x(t ; w)\right\}=e^{-A(w) t}(d / d t) x(t ; w)-A(w) e^{-A(w) t} x(t ; w)$

Therefore

$$
(d / d t)\left\{e^{-A(w) t} x(t ; w)\right\}=e^{-A(w) t} f(t, x(t ; w))
$$

Integrating both sides of Equation (6.2) from $t_{0}$ to $t$ we have

$$
e^{-A(w) t} x(t ; w)-e^{-A(w) t_{0}} x\left(t_{0} ; w\right)=\int_{t_{0}}^{t} e^{-A(w) \tau} f((\tau, x(\tau ; w)) d \tau
$$

Multiplying Equation (6.2) by $e^{A(w) t}$ and letting $t_{0}=0$, it reduces to

$$
x(t ; w)=e^{A(w) t} x_{0}(w)+\int_{0}^{t} e^{A(w)(t-\tau)} f((\tau, x(\tau ; w)) d \tau
$$


where $x_{0}(w)=x(0 ; w)$. Hence; if we let

$h(t ; w)=e^{A(w) t} x_{0}(w)$ and $k(t, \tau ; w)=e^{A(w)(t-\tau)}, 0 \leq \tau \leq t<\infty$

Equation (6.4) can be written as

$$
x(t ; w)=h(t ; w)+\int_{0}^{t} k(t, \tau ; w) f(\tau, x(\tau ; w)) d \tau
$$

Hence the stochastic differential system (6.1) reduces to the stochastic integral Equation (6.4), which is a special form of Equation (1.1).

Now we state the following theorem.

Theorem (6.1) Let us assume that the following conditions hold with respect to the stochastic integral Equation (6.4)

1) The matrix $A(w)$ is stochastically stable, that is there exists an $\alpha>0$ such that

$$
P\left\{w ; \operatorname{Re} \Psi_{k}(w)<-\alpha, k=1,2, \cdots, n\right\}=1
$$

where $\Psi_{k}(w), k=1,2, \cdots, n$ are the characteristic roots of the matrix.

2) $f(t, x)$ is a continuous function from $\mathbb{R}_{+} \times \mathbb{R}^{n} \rightarrow \mathbb{R}^{n}$ such that

$$
|f(t, x)-f(t, y)| \leq \lambda|x-y|
$$

With $f(t, 0)=0$ and $\lambda$ sufficiently small.

Then there exists a unique random solution of the stochastic integral Equation (6.4) such that

$$
\lim _{t \rightarrow \infty}\left\{\int_{\Omega}|x(t ; w)|^{2} d P(w)\right\}^{\frac{1}{2}}=0
$$

Proof To prove this result we want to prove that the pair of Banach spaces $\left(C_{g}, C_{g}\right)$ is admissible under conditions 1) and 2) with $g(t)=e^{-\beta t}$, and then apply theorem 4.1.

Recall that the norm in the space $C_{g}\left(\mathbb{R}_{+}, L_{2}(\Omega, \mathcal{F}, P)\right)$ is defined by

$$
\|x(t ; w)\|_{C_{g}}=\sup _{t \in R}[1 / g(t)]\left\{\int_{\Omega}|x(t ; w)|^{2} d P(w)\right\}^{\frac{1}{2}}
$$

and for any function $x(t ; w) \in C_{g}\left(\mathbb{R}_{+}, L_{2}(\Omega, \mathcal{F}, P)\right)$, let us define the following integral operator

$$
(T x)(t ; w)=\int_{0}^{t} k(t, \tau ; w) x(\tau ; w) d \tau
$$

Since $k(t, \tau ; w)=e^{A(w)(t-\tau)}, 0 \leq \tau \leq t<\infty$ Equation (6.5) becomes

$$
(T x)(t ; w)=\int_{0}^{t} e^{A(w)(t-\tau)} x(\tau ; w) d \tau
$$

or

$$
\|(T x)(t ; w)\| \leq \int_{0}^{t}\left\|e^{A(w)(t-\tau)}\right\|\|x(\tau ; w)\| d \tau
$$

It has been shown by Morozan [2] [3] that there exists a subset $D$ of $\Omega$ such that $P(D)=1$ and

$$
\left\|e^{A(w)(t-\tau)}\right\| \leq k e^{-\alpha(t-\tau)}
$$

for $w \in D, k>0$ and $\alpha$ as defined previously now, putting (6.7) into in equality (6.6) we have

$$
\|(T x)(t ; w)\| \leq k \int_{0}^{t} e^{-\alpha(t-\tau)}\|x(\tau ; w)\| d \tau \leq k e^{-\alpha(t-\tau)} \int_{0}^{t} e^{-\alpha(t-\tau)}[\|x(\tau ; w)\| / g(\tau)] g(\tau) d \tau
$$

Since $g(t)=e^{-\beta t}, 0<\beta<\alpha$, inequality (6.8) becomes

$$
\begin{aligned}
& \|(T x)(t ; w)\| \leq k \int_{0}^{t} e^{-\alpha(t-\tau)}\left(1 / e^{-\beta \tau}\right)\|x(\tau ; w)\| e^{-\beta \tau} d \tau \\
& \leq k\|x(t ; w)\|_{C_{g}} e^{-\alpha t} \int_{0}^{t} e^{\tau(\alpha-\beta)} d \tau \leq k\|x(t ; w)\|_{C_{g}}(\alpha-\beta)^{-1}\left(e^{-\beta t}-e^{-\alpha t}\right), t \geq 0
\end{aligned}
$$


Inequality (6.9) can be majorized as follows

$$
\|(T x)(t ; w)\| \leq k\|x(\tau ; w)\|_{C_{g}}(\alpha-\beta)^{-1} e^{-\beta t}
$$

Because $0<\beta<\alpha$. Dividing inequality (6.10) by $e^{-\beta t}$, we have

$$
\|(T x)(t ; w)\|_{C_{g}} \leq k(\alpha-\beta)^{-1}\|x(t ; w)\|_{C_{g}}
$$

Hence for $x(t ; w) \in C_{g}\left(\mathbb{R}_{+}, L_{2}(\Omega, \mathcal{F}, P)\right)$ we have $T C_{g}\left(\mathbb{R}_{+}, L_{2}(\Omega, \mathcal{F}, P)\right) \subset C_{g}\left(\mathbb{R}_{+}, L_{2}(\Omega, \mathcal{F}, P)\right)$ and the pair of Banach spaces $\left(C_{g}, C_{g}\right)$ is admissible.

The rest of the proof is due to theorem 4.1.

\subsection{A Problem in Telephone Traffic Theory}

In this subsection we shall examine a stochastic integral equation arising in the study of telephone traffic. We shall describe the problem in detail and then apply corollary 4.3 to show existence of a unique random solution

Consider a telephone exchange and suppose that calls arrive at the exchange at time instants $t_{1}, t_{2}, \cdots, t_{n}, \cdots$, where $0<t_{1}<t_{2}<\cdots<t_{n}<\infty$.

These arrival times must be considered as random instants, so we denote the distribution function by $A(t)$ on the time axis. For a call arriving at time $t$ let the random variable $H(t ; w)$ denote the holding time that is the length of time that a "conversation" is held for a call arriving at the exchange time $t$.

The $H_{1}(t ; w), H_{2}(t ; w), \cdots$ are considered as being mutually independent for different times $t_{1}, t_{2}, \cdots$ and as being independent of the state of the exchange, where the state of the exchange is the number of busy channels.

The number $m$ of trunks or channels of the exchange is assumed to be finite and large, so that we approximate a continuous process. It is also assumed that any channel not being used may be utilized by an incoming call and that the holding time for a channel beings at the time instant that the call arrives at the exchange.

A conversation (or connection) is realized if a channel is not busy at the time a call arrives. If all channels are busy at the time $t$ that a call arrives, then either the call is lost or a queueing problem develops. Only the first case will be considered here. Various problems have been studied in this situation. For example, the probability $P_{k}(t)$ that at time $t, k$ of the $m$ channels or busy has been examined in detail (Fortet [1]). We are concerned with the total number of "conversations" held (the number of busy channels) at time $t$ which for each $t$ is a random variable and may be described by as to chastic integral equation.

Let $x(t ; w)$ be the total number of conversations held at time $t$. That is $x(t ; w)$ is a random variable for each $t \in \mathbb{R}_{+}$and $x(0 ; w)=0$. Let $J(t ; w)$ be a random function with value one if a call arising at time $t>0$ is not lost and value zero if the call is lost

Let

$$
k(t, \tau ; w)= \begin{cases}1 & \text { if } t-\tau \in[0, H(\tau ; w)] ; \\ 0 & \text { if } t-\tau \notin[0, H(\tau ; w)] ;\end{cases}
$$

Such that $k(t, \tau ; w)$ is equal to one if a conversation from a call arising at time $\tau$ is still being held at time $t \geq \tau$ and is equal to zero otherwise. Thus we may write

$$
x(\tau ; w)=\int_{0}^{t} J(\tau ; w) k(t, \tau ; w) d A(\tau)
$$

Equation (6.11) is interpreted as the total number of telephone calls arising at times $\tau, 0 \leq \tau \leq t$ that were not lost such that the conversation is still being held at time $t$.

Suppose that $V(k)$ is any function such that

$$
V(k)=\left\{\begin{array}{cc}
1 & \text { if } k=0,1, \cdots, m-1 \\
0 & \text { otherwise }
\end{array}\right.
$$

Clearly, $x(t ; w) \leq m$ for all $t \in \mathbb{R}_{+}$and $w \in \Omega$. Hence we may write

$$
V[x(t ; w)]=\left\{\begin{array}{cc}
1 & \text { if } x(t ; w)=0,1, \cdots, m-1, \\
0 & \text { otherwise }
\end{array}\right.
$$


Which means that $V[x(t ; w)]$ has values one if a call arising at time $t$ is not lost and values zero otherwise. Then Equation (6.11) may be written as the nonlinear stochastic integral equation

$$
x(t ; w)=\int_{0}^{t} k(t, \tau ; w) v[x(\tau ; w)] d A(\tau)
$$

Which Bharucha-Reid [7] refers to as the Fortet integral equation.

Suppose that the distribution $A(t)$ of arrival times has a density function $a(t)$. Then Equation (6.12) reduces to a stochastic integral equation of the voltera type

$$
x(t ; w)=\int_{0}^{t} k(t, \tau ; w) v[x(\tau ; w)] a(\tau) d(\tau)
$$

If we let

$$
f(\tau, x(\tau ; w))=v[x(\tau ; w)] a(\tau)\left\{\begin{array}{cc}
a(\tau) & \text { if } x(\tau ; w)=0,1, \cdots, m-1 \\
0 & \text { otherwise }
\end{array}\right.
$$

Then we obtain a stochastic volterra integral equation of the form of (1.1) with the stochastic free term identically zero

$$
x(t ; w)=\int_{0}^{t} k(t, \tau ; w) f(\tau, x(\tau ; w)) d(\tau)
$$

$k(t, \tau ; w)$ is the stochastic kernel defined for $0 \leq \tau \leq t<\infty$ and taking only the value one or zero.

Before showing that (6.13) possesses a unique random solution we observe that the above description applies to many systems. If we replace the word "telephone exchange" with "serving mechanism" and the words "channel”, "call”, and "conversation" with the words "server", "customer”, and "service”, respectively, then we are dealing with a general system in which "customers" are being "served" by $m<\infty$ "servers". If we assume that a customer does not wait when he finds all $m$ servers busy so that no queue develops, then the random solution of the stochastic integral equation (6.13) gives the total number of "services" being performed at time $t$. Also the functions in (6.13) may be any functions describe the physical situation. For example the stochastic kernel may be of the form

$$
k(t, \tau ; w)=I_{X(w)}(t-\tau) e^{-(t-\tau)}
$$

where $I_{X(w)}(\cdot)$ is the indicator function of a random set $X(w)$, which means that solutions at earlier times $\tau \leq t$ have a decaying effect on the system.

We now show that the stochastic integral Equation (6.13) satisfies the conditions of corollary 4.3. We first show that

$$
f(t, x(t ; w)) \in L_{2}\left(\Omega, \mathcal{F} \text {, and } k(t, \tau ; w) \in L_{\infty}(\Omega, \mathcal{F}, P) P\right)
$$

Let $t \geq 0$ be fixed. Since $a(t)$ is a density function, it may be assumed to be bounded for all $t$ except on a set of measure zero. Hence for some $M>0$ and all $t, 0 \leq a(t)<\infty$ we have

$$
\int_{\Omega}|f(t, x(t ; w))|^{2} d P(w) \leq \int_{\Omega}|a(t)|^{2} d P(w) \leq M^{2}<\infty
$$

by definition of $f(t, x(t ; w))$, so that $f(t, x(t ; w)) \in L_{2}(\Omega, \mathcal{F}, P)$ for each $t \in \mathbb{R}_{+}$.

By definition of $k(t, \tau ; w), \quad 0 \leq \tau \leq t<\infty$, we obviously have that the $P$-measure of

$$
\{w:|k(t, \tau ; w)|>1\}, 0 \leq \tau \leq t<\infty
$$

is zero, that is a $P$-null set. Hence $k(t, \tau ; w)$ is bounded $P$-a.e. and is in $L_{\infty}(\Omega, \mathcal{F}, P)$. Also if $\left(t_{n}, \tau_{n},\right) \rightarrow(t, \tau)$ as $n \rightarrow \infty$ we have

$$
P\left\{w:\left|k\left(t_{n}, \tau_{n} ; w\right)-k(t, \tau ; w)\right|>0\right\} \rightarrow 0 \text { as } n \rightarrow \infty
$$

Since $k(t, \tau ; w)$ has values zero ore one only.

That $x(t ; w) \in C$, a continuous bounded function for each $w$, is easily shown. Let $w \in \Omega$ and choose $t \in \mathbb{R}_{+}$. For $\varepsilon>0$ and $h>0$ 


$$
|x(t+h ; w)-x(t ; w)|=\int_{t}^{t+h}|k(t, \tau ; w) f(\tau, x(\tau ; w)) d \tau| \leq\left|\int_{t}^{t+h} a(\tau) d \tau\right|
$$

Thus for $\varepsilon>0$ there is $a \delta>0$ such that when $|(t+h)-t|<\delta$

$$
\int_{t}^{t+h} a(\tau) d \tau<\varepsilon
$$

Therefore $x(t ; w)$ is continuous on $\mathbb{R}_{+}$for each $w \in \Omega$ and bounded by $m$ also

$$
\int_{\Omega}|x(t ; w)|^{2} d P(w) \leq m^{2}<\infty \quad t \in \mathbb{R}_{+}
$$

Which means that $x(t ; w) \in L_{2}(\Omega, \mathcal{F}, P)$ for each $t$.

We note that $\Lambda_{1}=1$ in corollary 4.3 since

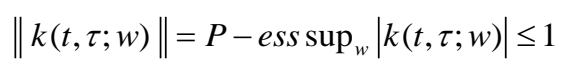

Since $h(t ; w) \equiv 0 \quad P$. a.e, trivially, it belongs to $C$. Since $a(t)$ is a density function, $f(t, 0)=a(t)$ is continuous, bounded, and nonnegative, and

$$
\int_{-\infty}^{\infty} a(t) d t<\infty
$$

Suppose $g(t)$ is any function satisfying condition 1) of corollary 4.3.

Then we must have

$$
|f(t, x)-f(t, y)|=\left\{\begin{array}{cc}
0 \leq \lambda g(t) \cdot 0 & \text { if } x=y \\
0 \leq \lambda g(t)(m-1) & \text { if } x \neq y ; x, y \in\{0,1, \cdots, m-1\} \\
a(t) \leq \lambda g(t) m & \text { if } x \neq m \text { and } y=m \text { or } x=m \text { and } y \neq m
\end{array}\right.
$$

If we choose $\lambda=1 / \mathrm{m}$, then in order for condition 2) of corollary 4.3 to be satisfied, there must exist appositive continuous function $g(t)$ on $\mathbb{R}_{+}$such that

$$
\int_{0}^{\infty} g(t) d t<\infty \text { and } a(t) \leq g(t) \quad t \in \mathbb{R}_{+}
$$

This restriction is not too sever, since $a(t)$ is a density function. We may take $g(t)=e^{-q t}, q>0$, for example. Therefore, since $\|h(t, w)\|_{C}=0,\|f(t, 0)\|=a(t) \leq M$ and $\lambda=1 / m$ is small for large $m$ there exist a unique random solution of Equation (6.13) if $a(t) \leq g(t)$, where $g(t)$ satisfies the given conditions.

\section{References}

[1] Tsokos, C.P. (1969) On a Monlinear Differential System with a Random Parameter. International Conference on System Sciences, IEEE Proceedings, Honolulu, Hawaii.

[2] Tsokos, C.P. (1969) On Some Nonlinear Differential System with Random Parameters. IEEE Proceedings Ann. Princeton Conf. on Information Sci. and Systems, 3rd, 228-234.

[3] Tsokos, C.P. (1969) On the Classical Stability Theorem of Poincare-Lyapunov. Proceedings of the Japan Academy, 45 , 780-785. http://dx.doi.org/10.3792/pja/1195520593

[4] Tsokos, C.P. (1969) On the Stochastic Integral Equation of the Volterra Type. Mathematical Systems Theory, 3, 222-231. http://dx.doi.org/10.1007/BF01703921

[5] Fortet, R. (1956) Random Distributions with Application to Telephone Engineering. Proc. Berkeley Symp. Math. Statist and Probability, 3rd, 11, 81-88.

[6] Bharucha-Reid, A.T. (1972) Random Integral Equations. Academic Press, New York.

[7] Corduneanu, C. (1968) Admissibility with Respect to an Integral Operator and Applications. Math. Tech. Rep., Univ. of Rhode Island.

[8] Tserpes, N.A. and Mukherjea, A. (1971) Invariant Measures on Semigroups with Closed Translations. Z. Wahr scheinlichk eits-theorie verw. Geb., 17, 33-38. http://dx.doi.org/10.1007/BF00538472

[9] Yosida, K. (1965) Functional Analysis. Springer-Verlag, Berlin and New York. 
[10] Adomian, G. (1970) Random Operator Equations in Mathematical Physics 1. Journal of Mathematical Physics, 11.

[11] Anderson, M.W. (1970) A Stochastic İntegral Equation. SIAM Journal on Applied Mathematics, 18, 526-532. http://dx.doi.org/10.1137/0118045 